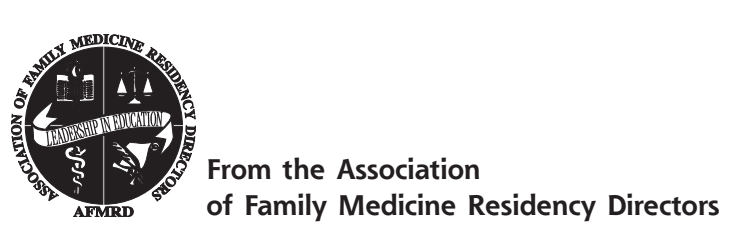

Ann Fam Med 2013;11:90. doi:10.1370/afm.1481.

\section{GME REFORM}

Reform is defined as "to improve by attention, correction of error, or removal of defects." Graduate Medical Education (GME) needs more attention, correction of errors, and removal of defects.

It is easy to point out the defects and errors of our graduate medical education system. We do not have enough physicians (some estimates predict a physician shortage of up to 159,000 by 2025); we do not have enough primary care physicians (by some estimates, we need at least $40 \%$ of physician force in primary care); our medical care system is too expensive $(\$ 9.6$ billion spent by Medicare for GME in 2010); or we are more focused on technology than patients (high tech versus high touch).

Is this sustainable? With the increasing number of our nation's seniors, we must ensure there are enough appropriately trained physicians to treat them. The Primary Care Residency Expansion Grant did increase primary care slots by 82 per year for 3 years, funded through HRSA. This program will produce only 246 additional primary care physicians. This should be an incentive to start at looking at alternative funding for GME positions rather than traditional Medicare sponsored cap positions. The Institute of Medicine (IOM) has authorized a GME Governance and Financing Committee to explore the production and cost of GME.

Congress is getting involved in the debate. In 2011, S 1627 (Nelson-Shurmer) bill was introduced to address resident physician shortage. S 3201 (KylReed) GME Reform Act of 2012 was introduced. HR 6352 (Schock-Schwartz) Resident Physician Shortage Reduction and GME Accountability and Transparency Act combines 1627 and 3201 .

HR 6352 has been endorsed by the American Academy of Medical Colleges (AAMC) and proposes to increase Medicare-reimbursed GME positions by 15,000 over 5 years $(2013-2017)$ but only $50 \%$ of the positions must be filled in a shortage specialty defined by the HRSA 2008 Workforce Report. Unfortunately, the report will not help primary care, as it used 1998 AMA data that shows family medicine, general internal medicine, and anesthesia in balance and general surgery, urology, ophthalmology, cardiology, orthopedics, and others as being in shortage. Hospitals over their cap could get more positions approved if training occurs in community health centers, community-based settings, or outpatient hospital departments. The concern for family medicine is that hospitals with less than 10 over their cap (most FM community hospitals) would not qualify for these positions. Unless the rules of this bill are changed to address the need for primary care, it will be difficult for family medicine organizations to support this bill. Family medicine needs individual physicians to contact House legislator offices to advocate for bill modification.

Regardless of legislative actions, GME reform must address critical questions/concerns. Why have ABFM board scores been decreasing over the past few years? Duty hour restrictions have been implemented for patient safety but have they had a negative impact on quality graduate medical education? There is an Accreditation Council for Graduate Medical Education (ACGME) length of training pilot to explore 4 vs 3 years of training in family medicine. Is longer training needed, or a significant alteration of the continuum of medical education? Do we still need 4 years of medical school training? Could the fourth year of medical school be enhanced to provide more substantive training? With the explosion of medical information, how can we enhance the training of our graduates in a resident friendly and patient safety environment? To ensure quality training, ACGME is requiring milestones which will describe satisfactory progress at set points throughout the residency. These will be debated and approved in 2013. The new revised RC-FM requirements will be out in 2013 or 2014; with input from AFMRD, hopefully these will define for our specialty the maternity requirements that have been discussed for the past few years.

GME reformation requires our attention and advocacy so that we can correct errors, remove defects, and produce physicians that provide high quality care in a cost effective manner for the needs of the American public.

Stoney Abercrombie, MDi, Todd Shaffer, MD, MBA Brian Crownover, MDi Grant Hoekzema, MDi Nathan Krug, MD, Lisa Maxwell, MD; Michael Mazzone, MD, Karen Mitchel, MD; Stephen Scbultz, MD, Michael Tuggy, MD 